\title{
Facile synthesis of liposome/ $\mathrm{Cu}_{2-x} \mathrm{~S}$-based nanocomposite for multimodal imaging and photothermal therapy
}

\author{
Juan Mou, Yu Chen, Ming Ma, Kun Zhang, Chenyang Wei, Hangrong Chen ${ }^{*}$ and Jianlin Shi*
}

\begin{abstract}
A kind of multifunctional perfluoropentane (PFP) and ultrasmall $\mathrm{Cu}_{2-\mathrm{x}} \mathrm{S}$ nanodots ( $\mathrm{u}-\mathrm{Cu}_{2-\mathrm{x}} \mathrm{S}$ NDs) co-incorporated liposome (PFP@ $\mathrm{u}-\mathrm{Cu}_{2-\mathrm{x}} \mathrm{S}$ NDs@liposome) nanocomposite has been facilely and successfully synthesized for enhanced ultrasound/infrared thermal/ photoacoustic multimodal imaging and photothermal therapy upon near infrared (NIR) laser irradiation. Such a liposome-based nanocomposite possesses a number of advantages, such as high dispersity and stability, excellent biocompatibility, small particle size $(<100$ $\mathrm{nm}$ ), well-defined core/shell structure, strong NIR absorption and photo-triggered vaporization of $\mathrm{PFP}$, etc. The detailed in vitro investigations demonstrate that the as-synthesized PFP@ u- $\mathrm{Cu}_{2-\mathrm{x}} \mathrm{S}$ NDs@ liposome nanocomposite is capable of enhancing the contrasts of ultrasound/infrared thermal/photoacoustic multimodal imaging, and substantially improving the photothermal therapeutic efficacy. This novel liposome-based theranostic nanoplatform shows great potentials in the future cancer diagnosis and therapy.
\end{abstract}

\section{INTRODUCTIDN}

Over the past decades, billions of people have suffered from various cancers worldwide [1]. Fortunately, diverse diagnostic imaging technologies [2-4] and powerful therapeutic methodologies [5-9] have emerged for cancer theranostics. As a minimally invasive and potentially effective therapeutic technique compared with conventional approaches, photothermal therapy (PTT) [10-13], which utilizes photothermal agents to convert near-infrared (NIR) energy into hyperthermia to kill cancer cells, has received extensive interest in recent years. Especially, imaging-guided PTT [14-17] shows great promise in improved efficiency and bio-safety.

Currently, ultrasound imaging [18-20] is believed to be one of the most widely used imaging tools in the clinic, owing to its considerably high resolution, deep tissue penetration, easy soft tissue recognition and low cost. However, the ultrasound waves are easily disrupted by air or gas and unable to penetrate bones [21]. Infrared thermal imaging [22-24] has recently attracted intensive interest thanks to its high imaging speed, sensitivity and convenience, but it suffers from relatively low imaging resolution. Recently, the newly developed photoacoustic imaging [25-28] is emerging as a complementary imaging modality, which combines the high contrast of optical imaging and the high spatial resolution of ultrasound imaging. Therefore, the combination of ultrasound/infrared theramal/photoacoustic imaging techniques will bring unexpected potentials in offering more comprehensive and accurate information with the complementary features of these techniques. In addition, the ingenious strategy of the multimodal imaging-guided PTT will be more powerful and efficient in PTT pre-guidance, intra-monitoring and post-evaluation. Therefore, the exploration of such multifunctional theranostic nanoplatforms, which combine both diagnostic and therapeutic functions to allow simultaneous multimodal imaging and therapy as well as the monitoring of tissue/organ's responses to the treatments, has been becoming a research hotspot in the current biomedicine filed.

The design of liposome-based nanocomposites [29-32] offered a great potential for the fabrication of such multifunctional theranostic nanoplatforms. Perfluorocarbons (PFCs) incorporated liposome [33-36] has long been being a common contrast agent for the ultrasound imaging by providing contrast enhancement through an acoustic impedance mismatch or resonance. Recently, a laser-induced phase transformation $[37,38]$ was proposed aiming at providing a safer and more controllable process of vaporization by using less energy than that of high-intensity ultrasound. Upon laser irradiation, a liquid-to-gas phase transformation of PFCs takes place due to the temperature elevation via the conversion of laser energy. The resulting PFCs bubbles are capable of enhancing contrast of ultrasound/ photoacoustic imaging $[39,40]$ and inducing cavitation for therapy [41-43] by the bubble formation and explosion. This kind of laser-induced phase transformation can be realized by the combination of PFCs droplets with various

State Key Laboratory of High Performance Ceramics and Superfine Microstructure, Shanghai Institute of Ceramics, Chinese Academy of Sciences, Shanghai 200050, China

*Corresponding authors (emails: hrchen@mail.sic.ac.cn (Chen H); jlshi@mail.sic.ac.cn (Shi J)) 
photosensitizers, such as indocyanine green (ICG)-loaded perfluorocarbon nanodroplets, gold nanoparticles and perfluorohexane liquid (PFH) co-loaded PLGA [42,43].

Herein, we propose a novel single NIR laser induced multifunctional theranostic nanoplatform for ultrasound/ infrared thermal/photoacoustic multimodal imaging and photothermal therapy, based on the rational design and construction of perfluoropropane (PFP) and ultrasmall $\mathrm{Cu}_{2-x} \mathrm{~S}$ nanodots ( $\mathrm{u}-\mathrm{Cu}_{2-x} \mathrm{~S}$ NDs) co-loaded liposome (abbreviated as PFP@u-Cu $\mathrm{Cu}_{2-x} \mathrm{~S}$ NDs@liposome). This multifunctional PFP@u-Cu $\mathrm{Cu}_{2-x} \mathrm{~S}$ NDs@liposome can be facilely synthesized via a typical thin film method, and demonstrates a number of excellent properties, including high dispersity and stability in aqueous solution, excellent biocompatibility, small particle size $(<100 \mathrm{~nm})$, well-defined core/shell structure, and strong NIR absorption originating from the copper deficient $\mathrm{u}-\mathrm{Cu}_{2-x} \mathrm{~S}$ NDs, which is attributed to the localized surface plasmon resonances [44-46]. In addition, PFP bubbles can be detected upon NIR laser irradiation. Therefore, the PFP@u-Cu $\mathrm{Cu}_{2-x} \mathrm{~S}$ NDs@liposome is demonstrated to be capable of providing significant contrast enhancements for ultrasound/infrared thermal/ photoacoustic multimodal imaging, and improving photothermal therapeutic efficacy for killing cancer cells in vitro. This novel NIR laser induced multifunctional theranostic nanoplatform based on PFP@u- $\mathrm{Cu}_{2-x} \mathrm{~S}$ NDs@liposome nanocomposite will attract extensive interest in future cancer diagnosis and therapy.

\section{RESULTS AND DISCUSSIONS}

The as-synthesized PFP and $\mathrm{u}-\mathrm{Cu}_{2-x} \mathrm{~S}$ NDs co-incorporated liposome (PFP@u-Cu $\mathrm{Cu}_{2-x} \mathrm{~S}$ NDs@liposome) for ultrasound/ infrared thermal/photoacoustic multimodal imaging and photothermal therapy is illustrated in Scheme 1. The novel PFP@u-Cu $\mathrm{Cu}_{2-x} \mathrm{~S}$ NDs@liposome was facilely and successfully synthesized by incorporating hydrophobic fluorocarbon compound PFP of low boiling point $\left(29^{\circ} \mathrm{C}\right)$ in the core and $\mathrm{u}-\mathrm{Cu}_{2-x} \mathrm{~S}$ NDs in the shell.

Blank liposome was prepared via a typical thin film method [47]. The representative transmission electron microscopy (TEM) image (Fig. 1a) of the as-synthesized blank liposome shows that the liposome is quasi-spherical with a multilamellar structure and a mean diameter of 100 $\mathrm{nm}$. This morphology offers high potential for the incorporation of PFP and $\mathrm{u}-\mathrm{Cu}_{2-x} \mathrm{~S}$ NDs with controllable bubbles release upon NIR laser irradiation. Moreover, the size of the liposome can be tailored in the synthetic process, ranging from several micrometers to dozens of nanometers, as shown in Fig. S1. The $\mathrm{u}-\mathrm{Cu}_{2-x} \mathrm{~S}$ NDs with a diameter of 2 $\mathrm{nm}$ (Fig. 1b) was synthesized as previously reported [48]. The typical scanning transmission electron microscopy (STEM) images (Figs 1c and inset) of PFP@u-Cu $\mathrm{Cu}_{2-x} \mathrm{~S}$ NDs@

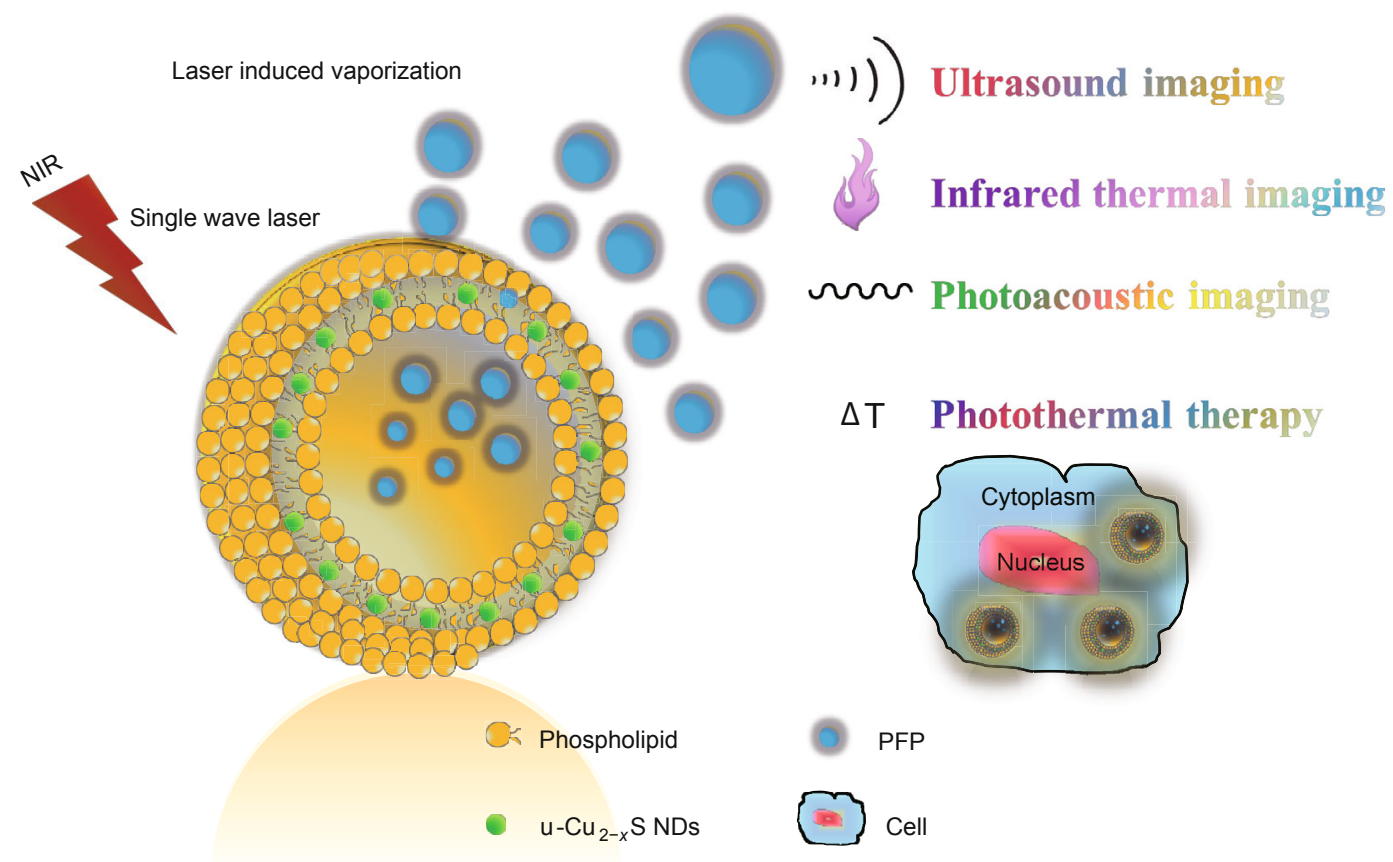

Scheme 1 Schematic illustration of the as-synthesized PFP@u-Cu $\mathrm{Cu}_{2-x} \mathrm{~S}$ NDs@liposome for enhanced ultrasound/infrared thermal/photoacoustic multimodal imaging and photothermal therapy under NIR laser irradiation. 

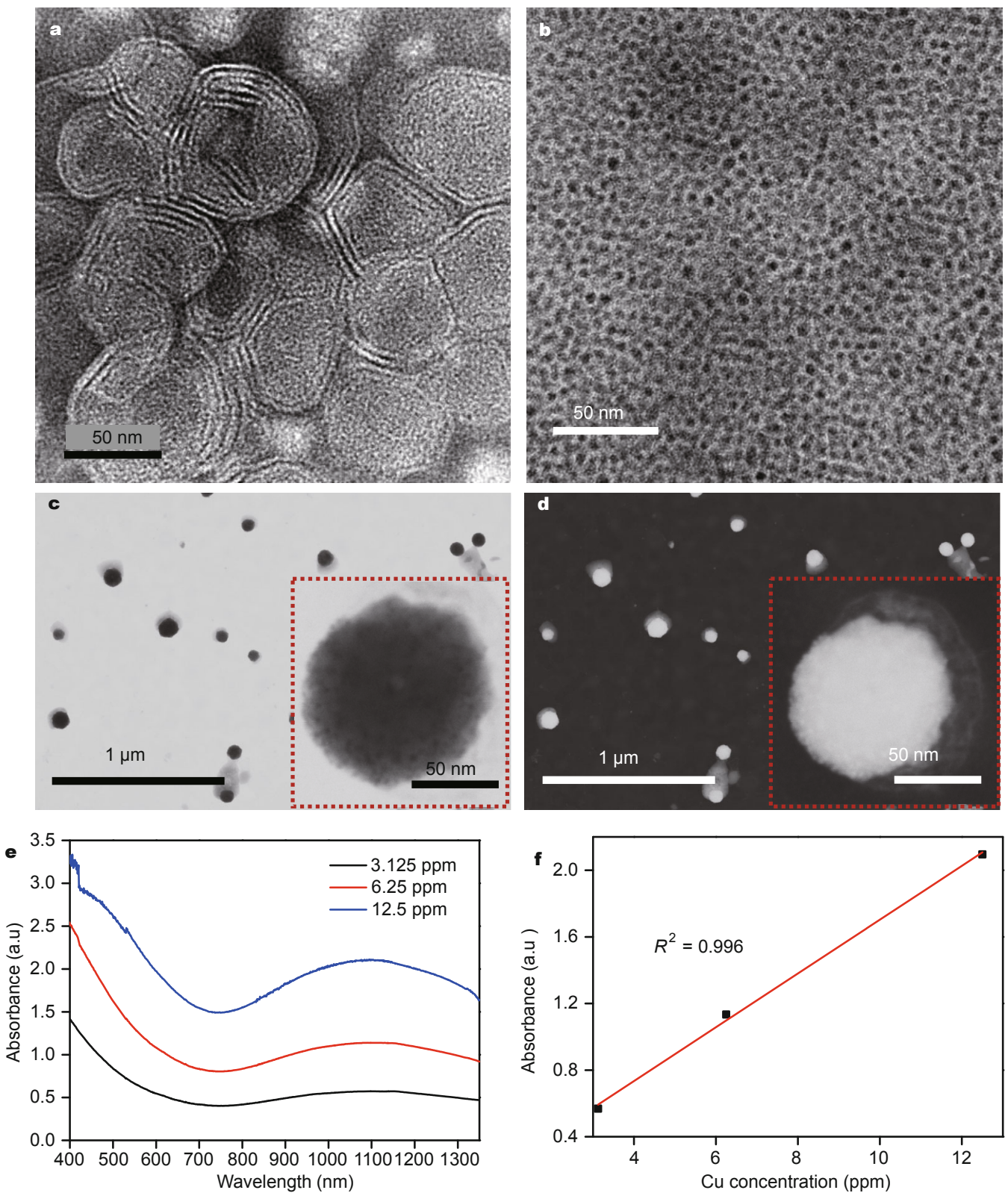

Figure 1 TEM images of blank liposome (a) and oleylamine-capped u- $\mathrm{Cu}_{2-x} \mathrm{~S}$ NDs (b). STEM (c) and HAADF-STEM image (d) of the PFP@u-Cu $\mathrm{u}_{2-x} \mathrm{~S}$ NDs@liposome. (e) UV-Vis absorption spectra of PFP@u- $\mathrm{Cu}_{2-x} \mathrm{~S}$ NDs@liposome dispersions at different $\mathrm{Cu}^{2+}$ concentrations and (f) the corresponding linear fitting plots of absorbance $v s . \mathrm{Cu}^{2+}$ concentration at $1064 \mathrm{~nm}$.

liposome show no significant morphology and size changes compared with blank liposome. The corresponding high angle annular dark field-STEM (HAADF-STEM) images (Figs 1d and inset) and elemental area mapping (Fig. S2) confirm the successful incorporation of $\mathrm{u}-\mathrm{Cu}_{2-x} \mathrm{~S}$ NDs. The hydrodynamic diameter of the PFP@u-Cu $\mathrm{Cu}_{2-x} \mathrm{~S}$ NDs@liposome was measured to be around $130 \mathrm{~nm}$ (Fig. S3a) and the Zeta potential was determined to be about $-13 \mathrm{mV}$ (Fig. $\mathrm{S} 3 \mathrm{~b})$. In addition, there was no change in the hydrodynamic diameter and Zeta potential after being kept at $4^{\circ} \mathrm{C}$ for 3 and 7 days, demonstrating the high stability of this multifunctional liposome nanoplatform. Moreover, it is worth noting that the as-synthesized PFP@u-Cu $\mathrm{Cu}_{2-x} \mathrm{~S}$ NDs@ liposome shows a broad and strong absorption from 800 to $1400 \mathrm{~nm}$ (Fig. 1e), with a peak locating in the second NIR window (1000-1350 nm). Additionally, the absorption intensity is linearly enhanced with increasing $\mathrm{Cu}^{2+}$ concentration (Fig. 1f), which is determined by inductively coupled plasma atomic emission spectroscopy (ICP-AES).

In order to distinctly demonstrate the successful in- 


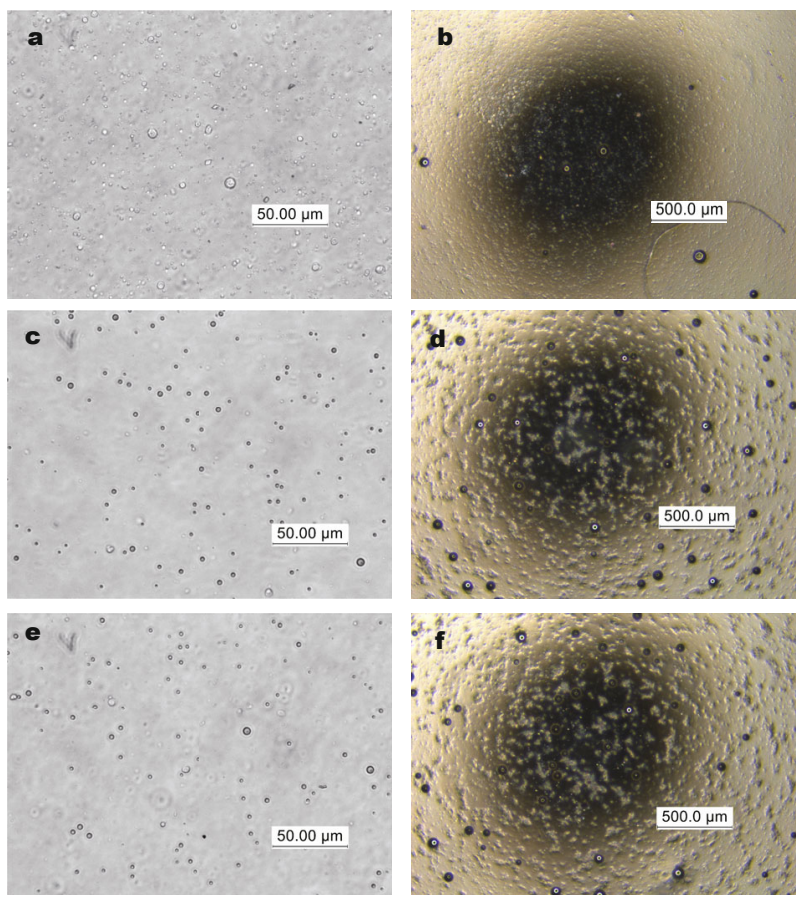

Figure 2 Optical microscopic images of $\mathrm{u}-\mathrm{Cu}_{2-x} \mathrm{~S}$ NDs@liposome (a, b) and PFP@u-Cu $\mathrm{Cu}_{2-x} \mathrm{~S}$ NDs@liposome dispersion immersed in hot water $\left(60^{\circ} \mathrm{C}\right)(\mathrm{c}, \mathrm{d})$, and exposed to NIR laser irradiation $\left(980 \mathrm{~nm}, 1.41 \mathrm{~W} \mathrm{~cm}^{-2}\right.$, $5 \mathrm{~min})(\mathrm{e}, \mathrm{f})$.

corporation of PFP in PFP@u-Cu $\mathrm{Cu}_{2-x} \mathrm{~S}$ NDs@liposome, the bubble generation assays in both hot water $\left(60^{\circ} \mathrm{C}\right)$ and by exposure to NIR laser irradiation were carried out. As shown in Figs $2 \mathrm{a}$ and $\mathrm{b}$, the reference $\mathrm{u}-\mathrm{Cu}_{2-x} \mathrm{~S}$ NDs@liposome sample without PFP shows no bubble generation both before and after being immersed in hot water. However, a great amount of smaller-sized phase-changed PFP micro-bubbles emerge in PFP@u- $\mathrm{Cu}_{2-x} \mathrm{~S}$ NDs@liposome dispersion after either being immersed in hot water (Figs 2c and d) or exposed to NIR laser irradiation (Figs 2e and $\mathrm{f}$ ), which undoubtedly confirms that both PFP and $\mathrm{u}-\mathrm{Cu}_{2-x} \mathrm{~S}$ NDs have been successfully incorporated into the liposome and great numbers of vaporized PFP nanoemulsions have been converted into gas bubbles. These small bubbles will further swell and merge into larger microbubbles, which is beneficial for enhanced ultrasound/photoacoustic imaging under NIR laser irradiation.

Encouraged by the strong NIR absorption of PFP@u$\mathrm{Cu}_{2-x} \mathrm{~S}$ NDs@liposome and sustained generation of PFP gas bubbles under NIR laser irradiation, we further investigated its contrast enhancement capability for ultrasound/ infrared thermal/photoacoustic multimodal imaging and photothermal effect in vitro. Two mililiter of PFP@liposome as a control and PFP@u- $\mathrm{Cu}_{2-x} \mathrm{~S}$ NDs@liposome dispersions $\left(\mathrm{Cu}^{2+}\right.$ concentration: $\left.50 \mathrm{ppm}\right)$ were wrapped up by a plastic tube and immersed into the degassed water tank for the following ultrasound imaging under NIR laser irradiation. The reference PFP@liposome dispersion shows no ultrasound imaging contrast enhancement after NIR laser irradiation (Figs 3a and b). Comparatively, a remarkable contrast enhancement (Figs $3 c$ and $d$ ) is observed in plastic tube containing the PFP@u-Cu $\mathrm{Cu}_{2-x} \mathrm{~S}$ NDs@liposome dispersion under exposure to NIR laser irradiation for 5 min (980 nm, power density: $1.41 \mathrm{~W} \mathrm{~cm}^{-2}$ ), which is attributed to the generation of large amount of PFP gas bubbles originated from the phase transformation of PFP. To further study the contrast enhancement capability of the PFP@u- $\mathrm{Cu}_{2-x} \mathrm{~S}$ NDs@liposome for infrared thermal imaging, infrared thermal images of reference water droplet and aqueous droplets containing the PFP@u-Cu $\mathrm{Cu}_{2-x} \mathrm{~S}$ NDs@ liposome ( $\mathrm{Cu}^{2+}$ concentration, $\left.\mathrm{C} 1: 12.5 \mathrm{ppm}, \mathrm{C} 2: 50 \mathrm{ppm}\right)$ at different time intervals were recorded during NIR laser irradiation for comparison. As shown in Figs 3e-h, water droplet shows no contrast enhancement and negligible temperature rise. In contrast, significant contrast enhanced infrared thermal images and temperature elevation are detected in the aqueous droplets containing the PFP@u-Cu $\mathrm{Cu}_{2-}$ ${ }_{x} \mathrm{~S}$ NDs@liposome under the same experimental conditions, indicating that this multifunctioal PFP@u- $\mathrm{Cu}_{2-x} \mathrm{~S}$ NDs@liposome is capable of enhancing the contrast of infrared thermal imaging. Furthermore, its potential of acting as a candidate contrast agent for photoacoustic imaging was also evaluated in vitro. Linear concentration-enhanced photoacoustic signals and images are obtained (Fig. 3i). In all, the PFP@u- $\mathrm{Cu}_{2-x} \mathrm{~S}$ NDs@liposome is demonstrated to be capable of substantially enhancing the contrasts of ultrasound/infrared thermal/photoacoustic multimodal imaging.

Moreover, the strong NIR absorption of PFP@u- $\mathrm{Cu}_{2-x} \mathrm{~S}$ NDs@liposome motivated us to investigate its photothermal effect. The temperature elevations of the aqueous dispersions containing PFP@u- $\mathrm{Cu}_{2-x} \mathrm{~S}$ NDs@liposome at different $\mathrm{Cu}^{2+}$ concentrations and under NIR laser irradiation at different power densities were measured. Pure water as a control demonstrated a temperature rise of $5^{\circ} \mathrm{C}$ under NIR laser irradiation with a power density of 1.41 $\mathrm{W} \mathrm{cm}{ }^{-2}$. Comparatively, the aqueous dispersions containing PFP@u-Cu $\mathrm{Cu}_{2-x} \mathrm{~S}$ NDs@liposome showed a temperature increase of $10-20^{\circ} \mathrm{C}$ in 5 min under the same experimental condition (Fig. 3j), and the heating rate increased with the increasing power density (Fig. S4). Thus, it is believed that the PFP@u-Cu $\mathrm{Cu}_{2-x} \mathrm{~S}$ NDs@liposome can efficiently and rapidly convert the $980 \mathrm{~nm}$ laser energy into hyperthermia, resulting from the strong absorption of $\mathrm{Cu}_{2-x} \mathrm{~S}$ NDs in NIR region. All these results demonstrate that this novel multifunctional PFP@u- $\mathrm{Cu}_{2-x} \mathrm{~S}$ NDs@liposome shows great potential in serving as an excellent contrast agent for ultrasound/infrared thermal/photoacoustic multimodal 

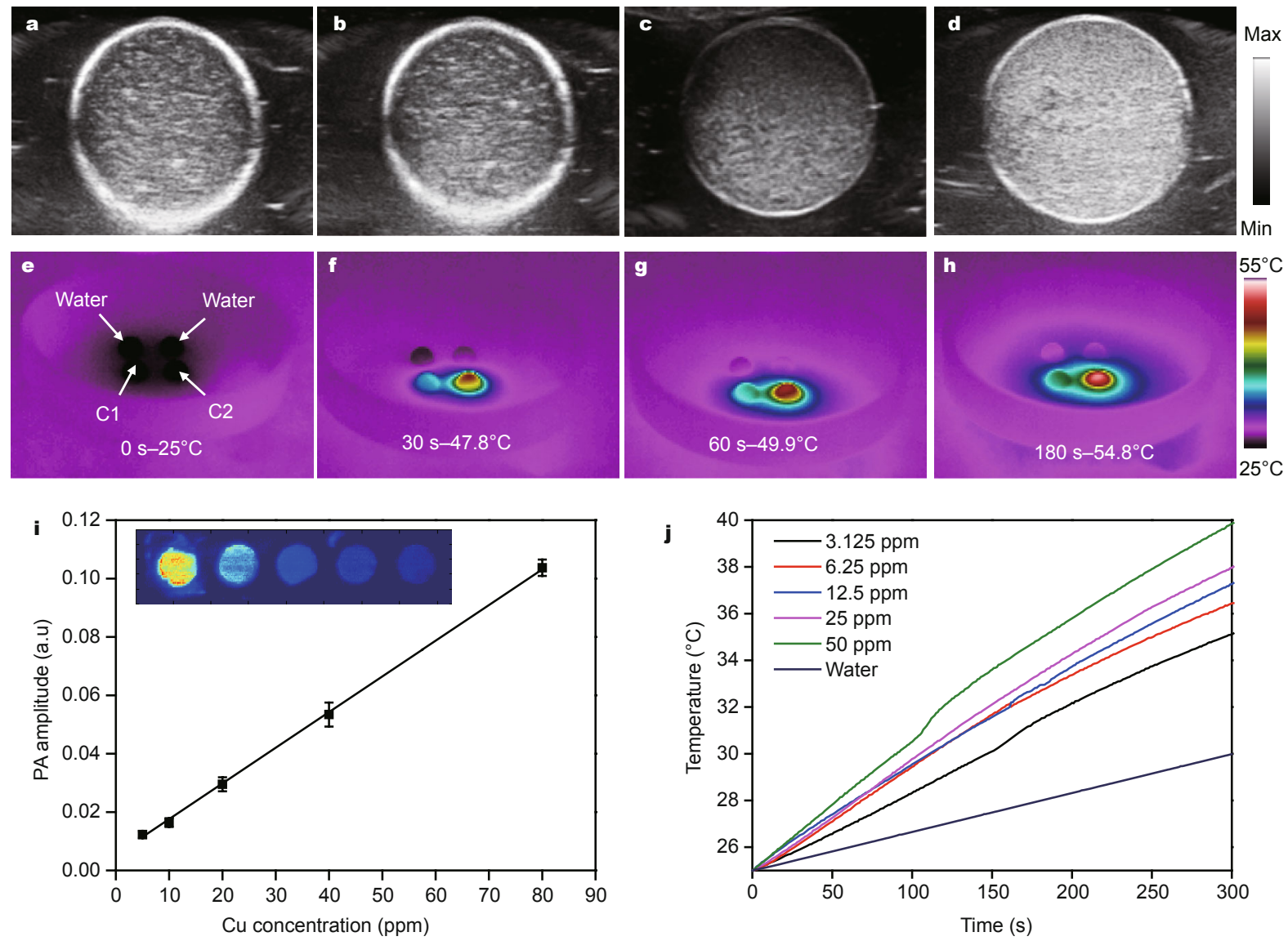

Figure 3 Ultrasound images of PFP@liposome (a, b) and PFP@ $\mathrm{u}-\mathrm{Cu}_{2-\mathrm{r}} \mathrm{S}$ NDs@liposome dispersion (c, d) before and after NIR laser irradiation for

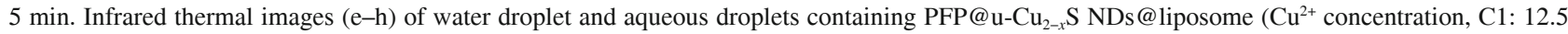
ppm, C2: $50 \mathrm{ppm}$ ) under NIR laser irradiation at different time intervals. (i) Linear plot of photoacoustic signal intensity $v s$. $\mathrm{Cu}^{2+}$ concentration, the inset shows the corresponding photoacoustic images of agar gel cylinders containing PFP@u-Cu $\mathrm{u}_{2-x} \mathrm{~S}$ NDs@liposome at varied Cu ${ }^{2+}$ concentrations. (j) Temperature elevations of the PFP@ $\mathrm{u}-\mathrm{Cu}_{2-x} \mathrm{~S}$ NDs@liposome dispersion exposed to NIR laser irradiation $\left(980 \mathrm{~nm}, 1.41 \mathrm{~W} \mathrm{~cm}^{-2}\right)$ vs. $\mathrm{Cu}^{2+} \mathrm{concentration}$

imaging and a highly efficient photothermal agent for photothermal therapy.

Furthermore, the cytotoxicity of the nanocomposite was evaluated by a standard 3-[4, 5-dimethylthiazol-2-yl]-2,5diphenyltetrazolium bromide (MTT) assay on HeLa cells. As expected, the PFP@u- $\mathrm{Cu}_{2-x} \mathrm{~S}$ NDs@liposome appears to be basically non-toxic. The cell viabilities are all above $90 \%$ even at a high $\mathrm{Cu}^{2+}$ concentration up to $100 \mathrm{ppm}$, as shown in Fig. S5 (the black histogram). Afterwards, the photothermal effect on HeLa cells was investigated. As demonstrated in Fig. S5 (the red histogram), the PFP@u- $\mathrm{Cu}_{2-x} \mathrm{~S}$ NDs@liposome exhibits a concentration-dependent cell death feature under NIR laser irradiation. In addition, the cell death was visualized by trypan blue staining in optical microscope. The control groups including HeLa cells without treatment, treated with the PFP@u-Cu $\mathrm{Cu}_{2-x} \mathrm{~S}$ NDs@liposome alone and NIR laser irradiation alone show no changes after trypan blue staining (Figs $4 \mathrm{a}-\mathrm{c}$ ). However, the cells treated with PFP@u- $\mathrm{Cu}_{2-x} \mathrm{~S}$ NDs@liposome and NIR laser irradiation are stained in blue, indicating that severe cell death (Fig. 4d) took place. Moreover, the cellular uptakes of the PFP@u-Cu $\mathrm{Cu}_{2-x} \mathrm{~S}$ NDs@liposome at varied duration were studied prior to confirming the cell death by confocal laser scanning microscopy (CLSM). It can be clearly found that most of the PFP@u-Cu $\mathrm{Cu}_{2-x} \mathrm{~S}$ NDs@liposome particles are in the cytoplasm of HeLa cells in $1 \mathrm{~h}$ (Fig. S6a) and 4 $\mathrm{h}$ (Fig. S6b) of co-incubation, demonstrating that the PF$\mathrm{P} @ \mathrm{u}-\mathrm{Cu}_{2-x} \mathrm{~S}$ NDs@liposome can be rapidly endocytosed by HeLa cells. The serve cell death was further verified by co-staining with calcein-AM and prodium iodide (PI) by CLSM. Similarly, the control cell groups mentioned above are stained in green after calcein-AM and PI co-staining, corresponding to live cells (Figs $4 \mathrm{e}-\mathrm{g}$ ). In contrast, the cells treated with the PFP@u-Cu $\mathrm{Cu}_{2-x} \mathrm{~S}$ NDs@liposome and NIR laser irradiation are stained in red, assigning to dead cells (Fig. 4h). These data are consistent with the results of MTT 

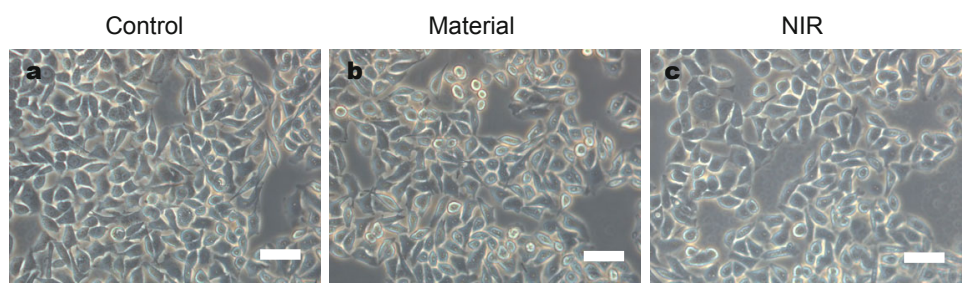

Material + NIR
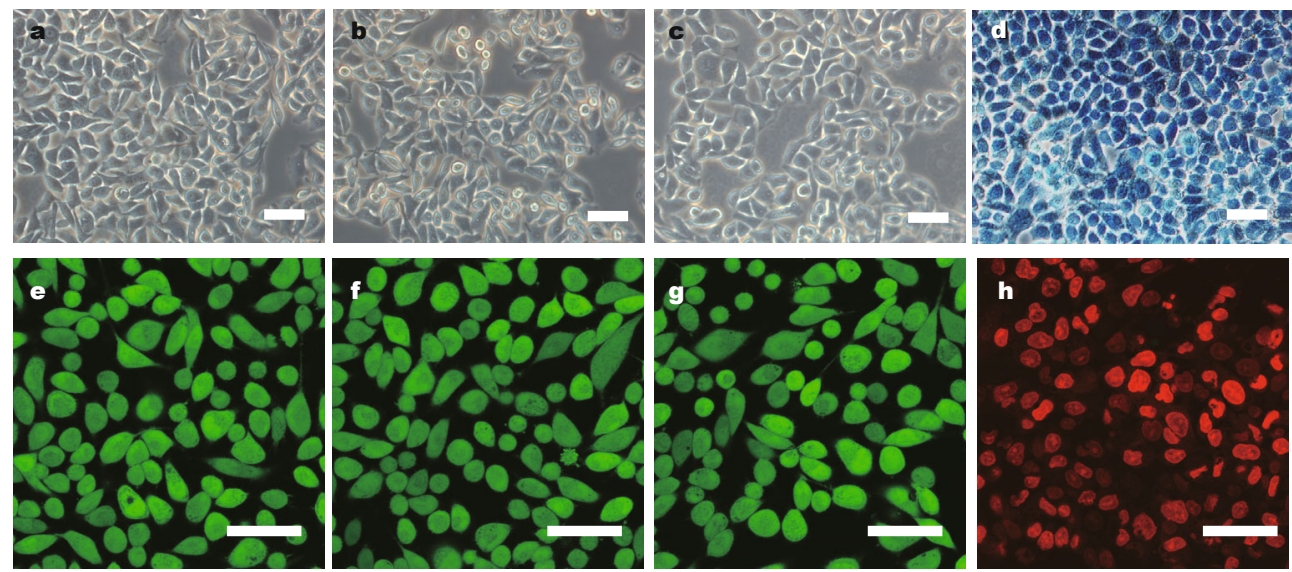

Figure 4 Optical microscopic images (a-d) and CLSM images (e-h) of HeLa cells stained with trypan blue, calcein-AM and prodium iodide (PI) after different treatments: (a, e) without treatment as a control; (b, f) treated with PFP@u- $\mathrm{Cu}_{2-x} \mathrm{~S}$ NDs@liposome alone; (c, g) treated with NIR laser irradiation alone; $(\mathrm{d}, \mathrm{h})$ treated with the PFP@ $\mathrm{u}-\mathrm{Cu}_{2-\mathrm{x}} \mathrm{S}$ NDs@liposome and NIR laser irradiation $\left(980 \mathrm{~nm}, 1.41 \mathrm{~W} \mathrm{~cm}^{-2}\right)$ together. All the scale bars in (a-h) are $50 \mu \mathrm{m}$.

assay and optical microscopic imaging. Therefore, it is believed that the PFP@u-Cu $\mathrm{Cu}_{2-x} \mathrm{~S}$ NDs@liposome is a highly effective photothermal agent to kill cancer cells in vitro, which is mainly attributed to the strong NIR absorption property, and therefore shows great potential in photothermal therapy in vivo.

\section{CONCLUSION}

In summary, a novel NIR laser induced multifunctional theranostic nanoplatform based on PFP@u-Cu $\mathrm{Cu}_{2-x} \mathrm{~S}$ NDs@liposome has been facilely and successfully fabricated. This kind of PFP@u-Cu ${ }_{2-x} \mathrm{~S}$ NDs@liposome nanocomposite demonstrates high dispersity and stability in aqueous solution, excellent biocompatibility; small particle size $(<100 \mathrm{~nm})$ and well-defined core/shell structure with PFP in the core and $\mathrm{u}-\mathrm{Cu}_{2-x} \mathrm{~S} \mathrm{NDs}$ in the shell. Specially, this nanocomposite exhibits strong absorption in the NIR region originating from unusual defect structure of $\mathrm{u}-\mathrm{Cu}_{2-x} \mathrm{~S}$ NDs. Therefore, this nanocomposite is capable of converting laser energy to hyperthermia to induce the phase transformation of PFP, resulting in sustained generation of gas bubbles. Under the same NIR laser irradiation, the PFP@u- $\mathrm{Cu}_{2-x} \mathrm{~S}$ NDs@ liposome is capable of generating significant contrast enhancement for ultrasound/infrared thermal/photoacoustic multimodal imaging and serving as an efficient photothermal agent for killing cancer cells. Thus, the as-synthesized PFP@u-Cu $\mathrm{Cu}_{2-x} \mathrm{~S}$ NDs@liposome will be a promising agent in multimodal imaging-guided photothermal therapy, which, as we believe, will also provide an ingenious strategy for the intensive exploration of liposome-based multifunctional nanomaterials in future in vivo cancer diagnosis and therapy.
Received 5 March 2015; accepted 3 April 2015 published online 10 April 2015

1 Siegel R, Ma J, Zou Z, et al. Cancer statistics, 2014. CA-Cancer J Clin, 2014, 64: 9-29

2 Fass L. Imaging and cancer: a review. Mol Oncol, 2008, 2: 115-152

3 Brody H. Medical imaging. Nature, 2013, 502: S81

4 Glasspool RM, Evans TRJ. Clinical imaging of cancer metastasis. Eur J Cancer, 2000, 36: 1661-1670

5 Manning MR, Cetas TC, Miller RC, et al. Clinical hyperthermia: results of a phase I trial employing hyperthermia alone or in combination with external beam or interstitial radiotherapy. Cancer, 1982, 49: 205-216

6 D’Amico AV, Whittington R, Malkowicz S, et al. Biochemical outcome after radical prostatectomy, external beam radiation therapy, or interstitial radiation therapy for clinically localized prostate cancer. JAMA, 1998, 280: 969-974

7 Nelson H, Petrelli N, Carlin A, et al. Guidelines 2000 for colon and rectal cancer surgery. J Natl Cancer Inst, 2001, 93: 583-596

8 Stewart LA. Chemotherapy in adult high-grade glioma: a systematic review and meta-analysis of individual patient data from 12 randomised trials. Lancet, 2002, 359: 1011-1018

9 Dougherty TJ, Gomer CJ, Henderson BW, et al. Photodynamic therapy. J Natl Cancer Inst, 1998, 90: 889-905

10 Huang X, El-Sayed IH, Qian W, et al. Cancer cell imaging and photothermal therapy in the near-infrared region by using gold nanorods. J Am Chem Soc, 2006, 128: 2115-2120

11 Lal S, Clare SE, Halas NJ. Nanoshell-enabled photothermal cancer therapy: impending clinical impact. Acc Chem Res, 2008, 41: 1842-1851

12 Gobin AM, Lee MH, Halas NJ, et al. Near-infrared resonant nanoshells for combined optical imaging and photothermal cancer therapy. Nano Lett, 2007, 7: 1929-1934

13 Loo C, Lowery A, Halas N, et al. Immunotargeted nanoshells for integrated cancer imaging and therapy. Nano Lett, 2005, 5: 709-711

14 Huang Y, He S, Cao W, et al. Biomedical nanomaterials for imaging-guided cancer therapy. Nanoscale, 2012, 4: 6135-6149

15 Yang $\mathrm{K}, \mathrm{Hu} \mathrm{L}, \mathrm{Ma} \mathrm{X}$, et al. Multimodal imaging guided photothermal therapy using functionalized graphene nanosheets anchored with magnetic nanoparticles. Adv Mater, 2012, 24: 1868-1872 
16 Zhou M, Zhang R, Huang M, et al. A chelator-free multifunctional $\left[{ }^{64} \mathrm{Cu}\right] \mathrm{CuS}$ nanoparticle platform for simultaneous micro-PET/CT imaging and photothermal ablation therapy. J Am Chem Soc, 2010, 132: 15351-15358

17 Jin Y, Li Y, Ma X, et al. Encapsulating tantalum oxide into polypyrrole nanoparticles for X-ray CT/photoacoustic bimodal imaging-guided photothermal ablation of cancer. Biomaterials, 2014, 35: 5795-5804

18 Deshpande N, Needles A, Willmann JK. Molecular ultrasound imaging: current status and future directions. Clin Radiol, 2010, 65: 567-581

19 Pignoli P, Tremoli E, Poli A, et al. Intimal plus medial thickness of the arterial wall: a direct measurement with ultrasound imaging. Circulation, 1986, 74: 1399-406

20 Fenster A, Downey DB. 3-D ultrasound imaging: a review. Eng Med Biol, IEEE, 1996, 15: 41-51

21 Herment A, Guglielmi JP, Dumee P, et al. Limitations of ultrasound imaging and image restoration. Ultrasonics, 1987, 25: 267-273

22 Sanchis-Sanchez E, Vergara-Hernandez C, Cibrian RM, et al. Infrared thermal imaging in the diagnosis of musculoskeletal injuries: a systematic review and meta-analysis. AJR Am J Roentgenol, 2014, 203: 875-882

23 Ring EF, Ammer K. Infrared thermal imaging in medicine. Physiol Meas, 2012, 33: R33-46

24 Lahiri BB, Bagavathiappan S, Jayakumar T, et al. Medical applications of infrared thermography: a review. Infrared Phys Technol, 2012, 55: 221-235

25 Wang LV, Hu S. Photoacoustic tomography: in vivo imaging from organelles to organs. Science, 2012, 335: 1458-1462

26 Wang X, Pang Y, Ku G, et al. Noninvasive laser-induced photoacoustic tomography for structural and functional in vivo imaging of the brain. Nat Biotechnol, 2003, 21: 803-806

27 Beard P. Biomedical photoacoustic imaging. Interface Focus, 2011, 1: 602-631

$28 \mathrm{Xu} \mathrm{M}$, Wang LV. Photoacoustic imaging in biomedicine. Rev Sci Instrum, 2006, 77: 041101

29 Martina MS, Fortin JP, Ménager C, et al. Generation of superparamagnetic liposomes revealed as highly efficient MRI contrast agents for in vivo imaging. J Am Chem Soc, 2005, 127: 10676-10685

30 Marie H, Lemaire L, Franconi F, et al. Superparamagnetic liposomes for MRI monitoring and external magnetic field-induced selective targeting of malignant brain tumors. Adv Funct Mater, 2015, 8: 1258-1269

31 Lozano N, Al-Jamal WT, Taruttis A, et al. Liposome-gold nanorod hybrids for high-resolution visualization deep in tissues. J Am Chem Soc, 2012, 134: 13256-13258

32 Langereis S, Keupp J, van Velthoven JLJ, et al. A temperature-sensitive liposomal ${ }^{1} \mathrm{H}$ CEST and ${ }^{19} \mathrm{~F}$ contrast agent for MR image-guided drug delivery. J Am Chem Soc, 2009, 131: 1380-1381

33 Hagisawa K, Nishioka T, Suzuki R, et al. Enhancement of ultrasonic thrombus imaging using novel liposomal bubbles targeting activated platelet glycoprotein IIb/IIIa complex-in vitro and in vivo study. Int J Cardiol, 152: 202-206

34 Hagisawa K, Nishioka T, Suzuki R, et al. Thrombus-targeted perfluorocarbon-containing liposomal bubbles for enhancement of ultrasonic thrombolysis: in vitro and in vivo study. J Thromb Haemost JTH, 2013, 11: 1565-1573

35 Strohm E, Rui M, Gorelikov I, et al. Vaporization of perfluorocar- bon droplets using optical irradiation. Biomed Opt Express, 2011, 2: $1432-1442$

36 Lindner JR. Microbubbles in medical imaging: current applications and future directions. Nat Rev Drug Discovery, 2004, 3: 527-533

37 Wilson K, Homan K, Emelianov S. Biomedical photoacoustics beyond thermal expansion using triggered nanodroplet vaporization for contrast-enhanced imaging. Nat Commun, 2012, 3: 618

38 Strohm E, Rui M, Gorelikov I, et al. Vaporization of perfluorocarbon droplets using optical irradiation. Biomed Opts express, 2011, 2: 1432-1442

39 Hannah AS, VanderLaan D, Chen YS, et al. Photoacoustic and ultrasound imaging using dual contrast perfluorocarbon nanodroplets triggered by laser pulses at $1064 \mathrm{~nm}$. Biomed Opt Express, 2014, 5: 3042-3052

40 Hannah A, Luke G, Wilson K, et al. Indocyanine green-loaded photoacoustic nanodroplets: dual contrast nanoconstructs for enhanced photoacoustic and ultrasound imaging. ACS Nano, 2013, 8: 250-259

$41 \mathrm{Ma} \mathrm{M}, \mathrm{Xu} \mathrm{H}$, Chen $\mathrm{H}$, et al. A drug-perfluorocarbon nanoemulsion with an ultrathin silica coating for the synergistic effect of chemotherapy and ablation by high-intensity focused ultrasound. Adv Mater, 2014, 26: 7378-7385

42 Zhou Y, Wang Z, Chen Y, et al. Microbubbles from gas-generating perfluorohexane nanoemulsions for targeted temperature-sensitive ultrasonography and synergistic HIFU ablation of tumors. Adv Mater, 2013, 25: 4123-4130

43 Sun Y, Wang Y, Niu C, et al. Laser-activatible PLGA microparticles for image-guided cancer therapy in vivo. Adv Funct Mater, 2014, 24: 7674-7680

44 Luther JM, Jain PK, Ewers T, et al. Localized surface plasmon resonances arising from free carriers in doped quantum dots. Nat Mater, 2011, 10: 361-366

45 Wei T, Liu Y, Dong W, et al. Surface-dependent localized surface plasmon resonances in CuS nanodisks. ACS Appl Mater Interfaces, 2013, 5: 10473-10477

46 Liu X, Swihart MT. Heavily-doped colloidal semiconductor and metal oxide nanocrystals: an emerging new class of plasmonic nanomaterials. Chem Soc Rev, 2014, 43: 3908-3920

47 Bangham AD, Standish MM, Watkins JC. Diffusion of univalent ions across the lamellae of swollen phospholipids. J Mol Biol, 1965, 13: 238-258

48 Mou J, Li P, Liu C, et al. Ultrasmall $\mathrm{Cu}_{2-x} \mathrm{~S}$ nanodots for highly efficient photoacoustic imaging-guided photothermal therapy. Small, doi: $10.1002 /$ smll.201403249

Acknowledgements This work was supported by the National Basic Research Program of China (2011CB707905), China National Funds for Distinguished Young Scientists (51225202), and Shanghai Excellent Academic Leaders Program (14XD1403800).

Author contributions Shi J and Chen $\mathrm{H}$ initiated and guided the work. Mou J designed and conducted the experiments. Chen Y, Ma M, Zhang K gave useful suggestions. Wei $\mathrm{C}$ set up the experimental devices.

Conflict of interest The authors declare that they have no conflict of interest.

Supplementary information Experimental details are available in the online version of the paper. 


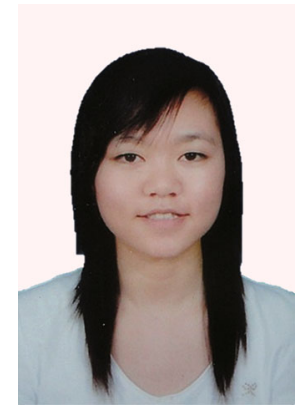

Juan Mou received her BSc degree at China University of Geosciences (Wuhan) (2007). She is now a PhD candidate at Shanghai Institute of Ceramics, Chinese Academy of Sciences (SICCAS). Her research interest includes the design, synthesis and biomedical applications of novel photothermal and photodynamic therapy materials.

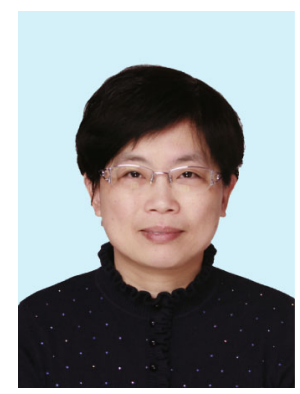

Hangrong Chen received her PhD degree in SICCAS (2001). She is now a professor of SICCAS. Her research areas include the synthesis of mesoporous materials, multifunctional inorganic biomedical nanomaterials, and novel environmental catalytic materials. She has published more than 150 scientific papers which have been cited more than 4600 times by other scientists with an h-index of 38 (2014).

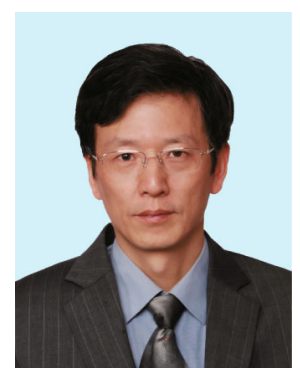

Jianlin Shi received his PhD degree in SICCAS (1989). He is now a professor of SICCAS. His research areas include the synthesis of mesoporous materials, mesoporous-based nano-composites, and their catalytic, biomedical and optical applications. He has published more than 300 scientific papers which have been cited more than 12,000 times by other scientists with an h-index of 59 (2014).

中文摘要 包裹氟碳化合物的脂质体已被广泛用作超声成像造影剂; 而硫化铜由于具有表面等离子体共振效应在近红外区域具有强 光学吸收特性, 可以同时作为一种性能优异的近红外热/光声成像造影剂和光热治疗剂. 本文以有机脂质体为载体, 同时包裏氟碳化合 物和硫化铜, 在近红外激光照射下, 硫化铜吸收光能将其转化为热能, 达到氟碳化合物的相变温度时使氟碳化合物由液态变为气态, 产 生的气泡可增强超声成像造影能力, 从而构建了单一波长激光诱导的超声/近红外热/光声多模式成像引导下的光热治疗, 极大提高了 癌症治疗的安全性和治疗效率. 体外实验结果表明该有机无机复合物不仅能克服单纯有机脂质体的不稳定性等缺点, 而且具有显著增 强的多模式成像造影能力和优异的光热治疗效果, 具有重要的临床应用前景. 